\title{
Co-occurring disorder assessment steps
}

Volume 4 Issue 3 - 2016

Keywords: Assessment process; Co-occurring disorders; Symptoms; Events; Diagnoses; Treatments

\section{Opinion}

Key components of the assessment process and how it plays a role in a client's treatment plan.

The foundation of an effective treatment plan begins with the assessment process. The counselor must gather as much detailed information pertaining to mental illness and substance abuse directly from the client as well as any family or friends that can provide insight. This assessment process can assist in finding a reliable diagnosis when devising a treatment plan. The counselor must ask the client to define what concerns they have about their mental health and/ or substance abuse issues. Through discussion the counselor can then identify what the client feels they need to change and how they would like to see these changes implemented into their lives.

The counselor must obtain information from the client arranged in order of occurrence to past symptoms, events, diagnoses, treatments and adversities that have played a substantial part in the client's mental health and substance abuse concerns. ${ }^{1}$

The counselor must acquire a comprehensive account of the client's strengths, limitations, support systems and cultural influences that must be considered when developing an individualized treatment plan.

The counselor and client both need to explore what stage of change they are currently in and if they are motivated to do the work to function as a whole in a healthier way. When the client is ready to make changes in their life they must have the tools, insight and resources necessary to help support them when they encounter unforeseeable challenges. Once they are able to reach identified goals the client and counselor can re-evaluate the treatment plan to address any new concerns. The stages of change can be unpredictable so the client must anticipate that they will encounter barriers, triggers and set-backs which may compromise their motivation for mental health or abstinence if they are not prepared. The counselor can assist the client in identifying the obstacles and help them identify all elements that will contribute to the success of the treatment plan. ${ }^{1}$

To ensure that the most accurate information is included in the treatment plan the counselor should follow a 12 step assessment process in order to obtain this information prior to developing a treatment plan. ${ }^{2}$

\section{i. Engage the client}

ii. After obtaining permission from the client and paperwork of the client authorizations the counselor can identify family and friends to gather any additional information that may be useful

iii. Screen for and detect Co-occurring disorders

iv. Determine the severity of mental and substance use disorders

\author{
Lisa Hold \\ CEO \& Founder, AllerGear, Washington D.C. Metro Area, USA \\ Correspondence: Lisa Hold, CEO \& Founder, AllerGear, \\ Washington D.C. Metro Area, USA,,Tel 5715998018, \\ Email lhold@allergear.com
}

Received: January 01, 1970 | Published: October 25, 2016

v. Determine an appropriate care setting for outpatient, inpatient or day treatment

vi. Determine the diagnoses

vii. Determine disability and functional impairments

viii. Identify strengths and supports of the client

ix. Identify the cultural and linguistic needs and supports

$\mathrm{x}$. Identify any additional problem areas to address such as housing, employment, education, social, physical health, spiritual and cognitive issues

\section{xi. Determine client's readiness for change}

\section{xii. Plan the treatment}

There are numerous methods that the counselor can use to gather data for the integrated assessment. For example, the counselor can interview the client, interview the client's family, interview friends, review psychiatric records, review medical records and physical examinations as well as order laboratory tests when developing a treatment plan. The information gathered should be used to help with the diagnosis of a client with co-occurring disorders. The diagnosis should not be based solely on the test but it gives a baseline to work with when developing an integrated treatment plan. Administering an appropriate assessment also helps the counselor focus on what areas need to be addressed when developing a treatment plan.

Individuals with co-occurring disorders are more complex than those presenting with one disorder and a reliable assessment instrument gives the counselor a map they can use when focus shifts from one concern to the next. The 12 step assessment process helps foster a good report with the client while at the same time gives the counselor a good idea of what issues the client needs to address.

\section{Acknowledgments}

None. 


\section{Conflicts of interest}

The authors declare there is no conflict of interests.

\section{Funding}

None.

\section{References}

1. Center for Substance Abuse Treatment. Substance abuse treatment for persons with co-occurring disorders. Treatment Improvement Protocol (TIP) Series 42. 2005.
2. Center for Substance Abuse Treatment. Substance abuse treatment: addressing the specific needs of women treatment improvement protocol (TIP) Series, No. 51.) Chapter 5: Treatment Engagement, Placement, and Planning. 2009. 